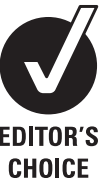

$10.1136 /$ ebn 1095

University of Tampa, Tampa, Florida, USA

Correspondence to: Cathy R Kessenich 401 W. Kennedy Blvd, Tampa, FL 33606, USA;

ckessenich@ut.edu

Randomised controlled trial

\title{
Administration of annual oral high-dose vitamin D to community dwelling older women in autumn and winter months increases risk of falls and fractures
}

\section{Cathy R Kessenich}

Commentary on: Sanders KM, Stuart AL, Williamson EJ, et al. Annual high-dose oral vitamin D and falls and fractures in older women: a randomized controlled trial. JAMA 2010;303:1815-22.

Vitamin D deficiency is an increasingly important worldwide issue. It is estimated that one billion people worldwide have vitamin D deficiency or insufficiency. ${ }^{1}$ Vitamin D deficiency has been linked to a multitude of problems across the lifespan. The importance of vitamin D and calcium absorption has been well documented. What remains unclear is the role of vitamin D in osteoporotic fracture prevention and specifically, the amount and regimen of vitamin D ingestion that is safe and effective in decreasing falls and subsequent fractures.

\section{Study details}

Sanders and colleagues conducted the largest randomised clinical trial to date of the effects of giving a year's supply of vitamin D in a single dose. The study hypothesis was reasonable in that vitamin D supplementation has been shown to reduce the risk of falls in community dwelling and institutionalised elders. ${ }^{2}{ }^{3}$ The intervention applied in this study was appealing in that compliance with daily or weekly supplementation is generally poor. Thus, annual dosing is an attractive option. The resulting increased risk of falls and fractures were surprising and counter to the results of most other studies of vitamin D supplementation. In giving 500000 IU once a year the researchers may have inadvertently exceeded the upper limit of vitamin D supplementation. The increased rate of falls and fractures experienced by the treatment group in this study raise questions about the value and safety of infrequent high doses of vitamin D.

\section{Additional explanations}

There is some evidence to suggest that vitamin D supplementation may be correlated to a reduction of musculoskeletal pain. ${ }^{4}$ The high dose of vitamin D used in this study may have served to reduce pain in the older subjects, leading to increased physical activity and subsequent falls and fractures. Other researchers have found that vitamin D supplementation reduced the incidence of wintertime bacterial and viral infections. ${ }^{56}$ Since the supplementation doses were given in the autumn and winter, women in the treatment group may have been protected against typical seasonal respiratory infections, more physically active and ultimately at greater risk for falls and fractures.

\section{Clinical impact}

Because vitamin D deficiency is increasingly recognised, many clinicians routinely measure serum vitamin D levels in patients of all ages. It has been common clinical practice to treat vitamin $\mathrm{D}$ deficient patients with loading doses of vitamin D (50000 IU once or twice weekly) for 6-8 weeks to bring serum levels back to nor- 
mal range. The study by Sanders and colleagues raises questions about the ultimate value of high doses of vitamin D supplementation. Vitamin D insufficiency is common and should be identified and corrected. However the risks and benefits of high doses given infrequently must be considered. There is currently no evidence of adverse effects of more frequent lower dose regimens. Clinicians should continue to identify vitamin D deficiency in patients. In those that are deficient, daily, weekly or monthly dosing may currently be the safest and most effective option.

\section{Gaps in evidence}

There is much to be learnt about vitamin D deficiency and the value of regular supplementation. Future studies should focus on the amount and scheduling of vitamin D ingestion necessary to avoid deleterious outcomes. Though the researchers found that an annual mega-dose of vitamin D was not effective, additional studies could show that the same amount given in smaller doses over a longer period of time may prevent falls and fractures.
Ultimately, the safety and efficacy of high-dose vitamin D supplementation requires further study.

\section{Competing interests None.}

\section{References}

1. Holick MF. Vitamin D deficiency. N Engl J Med 2007;357:266-81.

2. Bishchoff-Ferrari HA, Giovannuci E, Willett WC, et al. Estimation of optimal serum concentration of 25-hydroxyvitamin D for multiple health outcomes. Am J Clin Nutr 2006;84:18-28.

3. Broe KE, Chen TC, Weinberg J, et al. A higher dose of vitamin $\mathrm{d}$ reduces the risk of falls in nursing home residents: a randomized, multiple-dose study. J Am Geriatr Soc 2007;55:234-9.

4. Hicks GE, Shardell M, Miller RR, et al. Associations between vitamin D status and pain in older adults: the Invecchiare in Chianti study. J Am Geriatr Soc 2008;56:785-91.

5. Laaksi I, Ruohola JP, Tuohimaa P, et al. An association of serum vitamin D concentrations $<40 \mathrm{nmol} / \mathrm{L}$ with acute respiratory tract infection in young Finnish men. Am J Clin Nutr 2007;86:714-17.

6. Urashima M, Segawa T, Okazaki M, et al. Randomized trial of vitamin D supplementation to prevent seasonal influenza A in schoolchildren. Am J Clin Nutr 2010;91:1255-60. 